\title{
Tuning random lasers by engineered absorption
}

\author{
Ramy G. S. El-Dardiry a) and Ad Lagendijk \\ FOM-Institute for Atomic and Molecular Physics AMOLF, Science Park 104, 1098 XG Amsterdam, \\ The Netherlands
}

(Received 11 January 2011; accepted 22 February 2011; published online 19 April 2011)

\begin{abstract}
We achieve control over the emission wavelength of a random laser experimentally by adjusting the amount of absorption of emission light. The random laser consists of elastic scatterers in a high quantum efficiency gain medium to which a nonfluorescent dye is added. This nonfluorescent dye shifts the net gain curve and thereby the emission peak of the random laser. Mode competition effects are observed and explained. We interpret our results using a theoretical model that describes the spectral response of the system as a function of the effective cavity decay time and (re)absorption processes. (C) 2011 American Institute of Physics. [doi:10.1063/1.3571452]
\end{abstract}

Absorption and scattering of light are detrimental to most optical devices, since both processes lead to extinction of an incident light beam. ${ }^{1}$ In recent years however, disordered scattering media have been exploited to overcome fundamental limits of conventional optics. A combination of spatial speckle and uniform illumination has enabled sectioning of fluorescent images, ${ }^{2}$ spatial wavefront shaping has been applied to random media to improve the focus size, ${ }^{3}$ and designed disordered structures have been used to enhance the interaction between single emitters and single photons. ${ }^{4}$ These experimental studies emphasize that scattering is transforming from an annoyance into a tool. Furthermore, in most optics studies absorption is preferably minimized, for instance in the field of surface plasmon polaritons ${ }^{5}$ and in the field of random media.,

However, in the field of random lasing ${ }^{8-11}$ both absorption and multiple scattering of light are essential ingredients. In these special types of lasers, pump light is first absorbed and emission light is then generated inside a medium where multiple scattering of light acts as a feedback mechanism for stimulated emission of radiation. The gain curve of the emission molecules is the main mode selection mechanism in a random laser. ${ }^{12,13}$ Above threshold most light is emitted close to the maximum of the gain curve. Better control over these emission properties of a random laser has been a central issue in the field. ${ }^{8,14-16}$

In a convincing series of recent experiments, Gottardo et al. ${ }^{17}$ have shown that Mie resonances can shift the peak wavelength of a random laser by a few line widths. These Mie resonances introduce a wavelength dependent diffusion constant. The diffuse analog of the cavity decay time will therefore become wavelength dependent as well, which enables control over the emission wavelength of a random laser. Here, we show that control over the amount of absorption at the emission wavelength provides an alternative and much easier route to tailor intrinsically disordered lasers.

Random laser samples were fabricated using a Rhodamine $640 \mathrm{P}(\mathrm{Rh} 640)$ solution in methanol as a gain medium and titania particles (R900, Du Pont, 1 vol \%) as elastic scatterers. We added different quantities of Quinaldine Blue (Qblue), a nonfluorescent dye [quantum efficiency $0.1 \%$ (Ref. 18)], in methanol to control the random laser

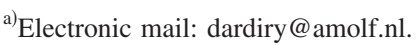

emission with absorption. The absorption and emission spectrum of both a $5 \mu \mathrm{M}$ solution of Rh640 and Qblue in methanol were measured using a UV-VIS spectrophotometer and a $535 \mathrm{~nm}$ pulsed optical parametric oscillator (Opolette, $20 \mathrm{~Hz}$, $5 \mathrm{~ns}, 535 \mathrm{~nm}$ ) respectively. These absorption and emission spectra are shown in Fig. 1. The emission cross section was calculated using $\sigma_{e}(\lambda)=\left(\lambda^{4} L(\lambda) / 8 \pi c \tau \eta^{2}\right)$. Here $\int L(\lambda) d \lambda$ $=\phi$ where $\phi$ is the quantum efficiency and $\eta$ is the effective refractive index. We assumed a radiative lifetime of $4 \mathrm{~ns}$ (Ref. 19) and a quantum efficiency of 1 for Rhodamine 640 P. ${ }^{13}$ The maximum absorption cross section of Qblue lies around the maximum emission cross section of Rh640, which makes Qblue very suited for engineering a Rh640 random laser. Moreover, Qblue has a stimulated emission cross section that is $\sim 100 \times$ smaller than the emission cross section of Rh640. Therefore, we can attribute all emission in the random laser experiments directly to emission originating from Rh640 molecules. We prepared four samples by combining a $1 \mathrm{mM}$ solution of Rh640 with a $1 \mathrm{mM}$ solution of Qblue such that $\rho_{\mathrm{Rh} 640}+\rho_{\mathrm{Qblue}}=1 \mathrm{mM}$ : sample $1 \quad(100 \%$ Rh640), sample 2 (96\% Rh640 and 4\% Qblue), sample 3 (90\% Rh640 and 10\% Qblue), and sample 4 (75\% Rh640 and $25 \%$ Qblue). A fifth sample was made by adding Qblue in crystalline form to a $1 \mathrm{mM}$ solution of Rh640 (molar ratio QBlue/Rh640=0.4). To prevent aggregation and sedimentation of titania particles all samples were treated in an ultrasonic bath before measurement.

Excitation light generated by the optical parametric oscillator was focused onto the samples by a lens $(f=5 \mathrm{~cm})$.



FIG. 1. (Color) Absorption (red line) and emission (red dashed line) spectra of Rhodamine $640 \mathrm{P}$ and absorption spectrum of Qblue (blue line) dissolved in methanol. 


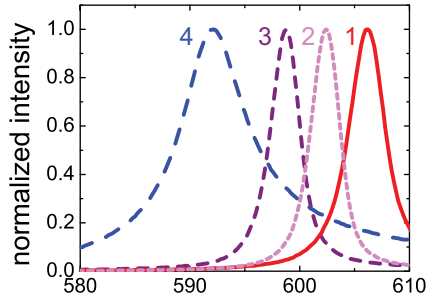

(a)

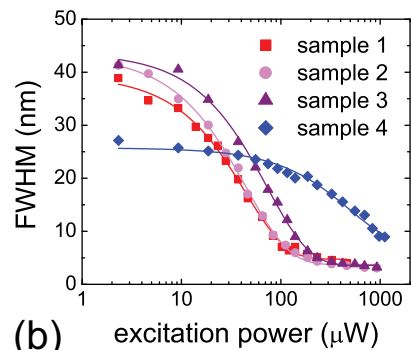

FIG. 2. (Color) (a) Emission spectrum above threshold for random laser samples 1-4. Pump powers: $465 \mu \mathrm{W}$ for sample 1, $930 \mu \mathrm{W}$ for sample 2 and 3, and $1114 \mu \mathrm{W}$ for sample 4. (b) Width of the spectrum vs pump power for random laser samples $1-4$.

The size of the fluorescent spot could be varied by moving the sample in and out of the focal plane. The same lens collected the emission which was then spectrally analyzed using a spectrograph (Oriel MS-257) connected to an electron-multiplying CCD camera (Hamamatsu, C-9100).

For all random laser samples, the emission spectrum was monitored for different values of the pump fluence. Above a certain pump value, that is the threshold, the emission spectrum narrows. We analyze the wavelength of the peak of the emission spectrum for the different samples. In Fig. 2(a) the spectra above threshold are plotted for the samples 1-4. The peak wavelength for a pure Rh640 random laser lies at 607 $\mathrm{nm}$ and shifts gradually to the blue when more Qblue molecules are added. When the ratio between Rh640 and Qblue molecules lies around 10:1 (sample 3), the peak is already shifted by about 2 line widths to $598 \mathrm{~nm}$. The spectrum of sample 4 is shifted by $14 \mathrm{~nm}$ compared to sample 1 , which corresponds to 3-4 line widths. The spectra of sample 1,2 , and 3 are significantly narrower than the spectrum of sample 4. To understand why the spectrum of sample 4 is relatively broad, it is insightful to plot the width of the spectra versus pump power as shown in Fig. 2(b). The widths were determined by the full-width at half maximum (FWHM) of the raw spectra, except for the first nine data points of sample 4 where two Lorentzians were fitted from the data from which the width was extracted. This fitting procedure was applied because these nine spectra consisted of two overlapping peaks. From this figure, we conclude that the threshold increases when the density of absorbers becomes larger. Sample 4, having the largest concentration of absorbers, has such a high threshold that the width is not yet converged to the far above threshold value at the maximum available input fluence.

When the concentration of the absorber is further increased, the random laser responses strikingly different. In Fig. 3(a), the normalized spectra below and above threshold are plotted for sample 5. Below threshold, two peaks can be observed in the emission spectrum: one around $590 \mathrm{~nm}$ and another lower one around $650 \mathrm{~nm}$. Above threshold, this situation is reversed: the peak at $650 \mathrm{~nm}$ dominates over the peak at $590 \mathrm{~nm}$. Figure 3(b) shows the peak spectral radiance for the two peaks versus excitation power. The pump power was increased monotonically to avoid irreversible artifacts. The ratio of the two peaks (peak at $590 \mathrm{~nm} /$ peak at $650 \mathrm{~nm}$ ) first increases with increasing pump power and then drops dramatically far above threshold. While the pump power increases, the peak emission wavelength changes significantly as shown in Fig. 4. The peak at the blue side of the spectrum


FIG. 3. (Color) (a) Spectra below (black, $84 \mu \mathrm{W}$ ) and above (gray, $2230 \mu \mathrm{W}$ ) threshold for sample 5. The absolute peak spectral radiance is a factor $\sim 30$ higher for the spectrum above threshold. Peak indicated by blue square: peak 1. Peak indicated by red disk: peak 2. (b) peak spectral radiance for the two peaks normalized to the data point at $4.6 \mu \mathrm{W}$ vs pump power. The black line is the ratio of the absolute values of the two peaks.

shows a blueshift of $3 \mathrm{~nm}$, whereas the peak at the red side of the spectrum shows a redshift of $9 \mathrm{~nm}$.

To describe and understand our experimental findings, we present an analytical expression for the peak wavelength of the random laser. In principle, for a constant mean cavity decay time and negligible depletion of the ground state, the peak wavelength of a random laser does not change as a function of pump power. Therefore, to describe the spectral response of the different random laser samples we study the steady-state solution of the rate equation for the photon density $q_{\omega}$ below threshold

$$
\begin{aligned}
& \frac{\mathrm{d} q_{\omega}}{\mathrm{d} t}=-\gamma_{c} q_{\omega}-c \rho_{1} \sigma_{\omega}^{a, 1} q_{\omega}-c \rho_{2} \sigma_{\omega}^{a, 2} q_{\omega}+\gamma_{r} n L_{\omega}, \\
& q_{\omega}=\left[\gamma_{c}+c \rho_{1} \sigma_{\omega}^{a, 1}+c \rho_{2} \sigma_{\omega}^{a, 2}\right]^{-1} \gamma_{r} n L_{\omega} .
\end{aligned}
$$

Here $n$ is the density of molecules in the upper laser level, $\gamma_{c}$ is the effective cavity decay rate ${ }^{20} \gamma_{r}$ is the radiative decay rate, $c$ is the speed of light, $\sigma_{\omega}^{a, 1}$ is the absorption cross section at frequency $\omega$ for the Rh640 molecules with density $\rho_{1}$, $\sigma_{\omega}^{a, 2}$ is the absorption cross section at frequency $\omega$ for the Qblue molecules with density $\rho_{2}$, and $L_{\omega}$ is the probability of a spontaneous emission event at frequency $\omega$. In the above equations we assume $n \ll \rho_{1}$, which is valid below threshold. Equation (2) describes how the spontaneous emission spectrum of a pure dye, $L_{\omega}$, is affected by the cavity decay rate and (re)absorption processes by the form factor $\left[\gamma_{c}\right.$ $\left.+c \rho_{1} \sigma_{\omega}^{a, 1}+c \rho_{2} \sigma_{\omega}^{a, 2}\right]^{-1}$.

The effective cavity decay time, $\gamma_{c}^{-1}$, can be approximated by the Thouless time, $\tau_{T}=(1 / 8)\left(L^{2} / D\right)$, which describes the average time a photon spends in a slab with dif-

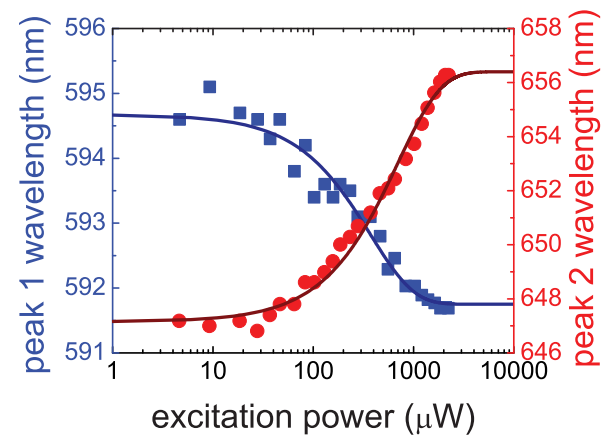

FIG. 4. (Color) Wavelength of peak emission vs pump power for sample 5 The peak at the blue side of the spectrum (left $y$-axis) shows a blueshift, whereas the peak at the red side of the spectrum (right $y$-axis) shows a redshift. The solid lines are guides to the eye. 


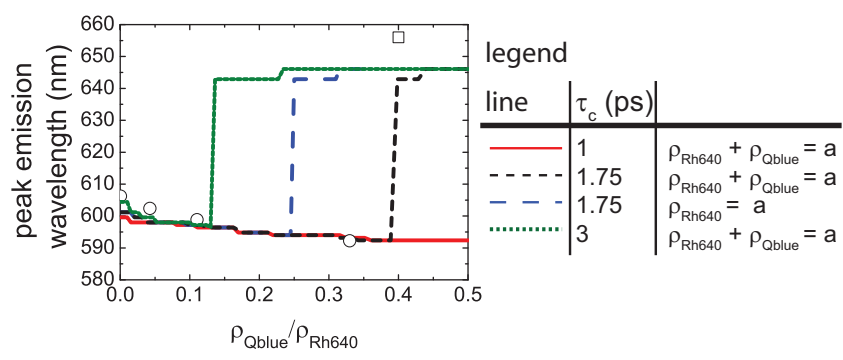

FIG. 5. (Color) Spectral position of the emission peak vs ratio between concentration of Qblue and Rh640 molecules. Black open circles: experimental data points for sample 1-4. Black open square: sample 5. Red, black, and green line: model with $\tau_{c}=1 \mathrm{ps}, 1.75 \mathrm{ps}$, and $3 \mathrm{ps}$, respectively, and $\rho_{\mathrm{Rh} 640}+\rho_{\mathrm{Qblue}}=1 \mathrm{mM}$. Blue dashed line: model with $\tau_{c}=1.75 \mathrm{ps}$ and $\rho_{\mathrm{Rh} 640}=1 \mathrm{mM}$.

fusion constant $D$ and thickness $L$. In our experimental configuration the exact value for the Thouless time is not known, since the excitation volume does not resemble a slab. If we assume the fluorescence FWHM to be the characteristic length scale $(\mathrm{FWHM} \approx 130 \mu \mathrm{m})$ and a transport mean free path of $\approx 5 \mu \mathrm{m}$, the Thouless time is on the order of picoseconds.

Figure 5 shows theoretical curves together with experimental data points for the peak position of the spectrum versus the ratio $\rho_{\text {Qblue }} / \rho_{\text {Rh640. }}$. For increasing concentrations of Qblue the peak emission shifts to the blue as is also clearly observed in experiment. The jumps in the theoretical curves show that for cavity decay times larger than $1 \mathrm{ps}$, the main peak in the spectrum changes from the blue part to the red part of the spectra for a certain concentration of Qblue. This dramatic change in peak position occurs for lower concentrations of Qblue when the cavity decay time is increased. This theoretical analysis enables us to understand the experimentally observed mode competition effect. In a previous experimental and numerical study, ${ }^{21}$ it was shown that around the random lasing threshold the emission volume expands. Such an expansion in volume leads to a larger mean cavity decay time and can thus lead to a spectral switch in the main random laser peak. Looking at the expression of the form factor in Eq. (2) suggests that a change in the concentration of molecules in the ground state also affects the form factor. However, such a depletion of the ground state cannot explain our experimental results since it would lead to less reabsorption and hence spectral shifts toward the gain maximum.

In conclusion, we have shown that introducing absorption leads to control over the peak emission wavelength of a random laser. A simple theoretical model enables to predict the position of the peak for various concentrations of the absorber. Our studies can be extended to absorbers other than nonfluorescent dyes, such as metal complexes and plasmonic metal nanoparticles, ${ }^{22,23}$ in order to increase the flexibility in the output of a random laser.

We thank Ronald Mooiweer and Timmo van der Beek for stimulating discussions. This work is part of the research program of the "Stichting voor Fundamenteel Onderzoek der Materie (FOM)," which is financially supported by the "Nederlandse Organisatie voor Wetenschappelijk Onderzoek (NWO)."

${ }^{1}$ H. C. van de Hulst, Light Scattering by Small Particles (Dover, New York, 1981).

${ }^{2}$ D. Lim, K. K. Chu, and J. Mertz, Opt. Lett. 33, 1819 (2008).

${ }^{3}$ I. M. Vellekoop, A. Lagendijk, and A. P. Mosk, Nat. Photonics 4, 320 (2010).

${ }^{4}$ L. Sapienza, H. Thyrrestrup, S. Stobbe, P. D. Garcia, S. Smolka, and P. Lodahl, Science 327, 1352 (2010).

${ }^{5}$ W. L. Barnes, A. Dereux, and T. W. Ebbesen, Nature (London) 424, 824 (2003).

${ }^{6}$ D. S. Wiersma, P. Bartolini, A. Lagendijk, and R. Righini, Nature (London) 390, 671 (1997)

${ }^{7}$ F. Scheffold, R. Lenke, R. Tweer, and G. Maret, Nature (London) 398, 206 (1999).

${ }^{8}$ M. A. Noginov, Solid-State Random Lasers (Springer, New York, 2005).

${ }^{9}$ H. Cao, J. Phys. A 38, 10497 (2005).

${ }^{10}$ D. S. Wiersma, Nat. Phys. 4, 359 (2008).

${ }^{11}$ M. Leonetti and C. Conti, J. Opt. Soc. Am. B 27, 1446 (2010).

${ }^{12}$ G. van Soest and A. Lagendijk, Phys. Rev. E 65, 047601 (2002)

${ }^{13}$ R. G. S. El-Dardiry, S. Faez, and A. Lagendijk, Phys. Rev. A 83, 031801(R) (2011).

${ }^{14}$ D. S. Wiersma and S. Cavalieri, Nature (London) 414, 708 (2001).

${ }^{15}$ E. Tikhonov, V. P. Yashchuk, O. Prygodjuk, and V. Bezrodny, Solid State Phenom. 106, 87 (2005).

${ }^{16}$ Y. Tian, X. Ma, P. Chen, Y. Zhang, and D. Yang, Opt. Express 18, 10668 (2010)

${ }^{17}$ S. Gottardo, R. Sapienza, P. D. García, A. Blanco, D. S. Wiersma, and C. López, Nat. Photonics 2, 429 (2008).

${ }^{18}$ S. Rentsch, R. Danielius, and R. Gadonas, J. Inf. Rec. Mater. 12, 319 (1984).

${ }^{19}$ N. M. Lawandy, R. M. Balachandran, A. S. L. Gomes, and E. Sauvain, Nature (London) 368, 436 (1994).

${ }^{20}$ K. L. van der Molen, A. P. Mosk, and A. Lagendijk, Phys. Rev. A 74, 053808 (2006).

${ }^{21}$ R. G. S. El-Dardiry, A. P. Mosk, and A. Lagendijk, Opt. Lett. 35, 3063 (2010)

${ }^{22}$ G. D. Dice, S. Mujumdar, and A. Y. Elezzabi, Appl. Phys. Lett. 86, 131105 (2005).

${ }^{23}$ X. Meng, K. Fujita, Y. Zong, S. Murai, and K. Tanaka, Appl. Phys. Lett. 92, 201112 (2008). 